\title{
"Total Mesenteric Peritonectomy for Peritoneal Metastases" (with Video) by Jean-Baptiste Cazauran et al.
}

\author{
Paul H. Sugarbaker, MD, FACS, FRCS \\ Center for Gastrointestinal Malignancies, MedStar Washington Hospital Center, Washington, DC
}

Early successes in the management of peritoneal metastases were based on preventing cancer implants that often resulted after the resection of a primary gastrointestinal cancer. The first reports of benefit, coming from Japan, involved gastric cancer with serosal invasion, ${ }^{1}$ or cytology-positive gastric cancer. ${ }^{2}$

The safest and most effective way to interrupt the progression of disease on peritoneal surfaces was treatment with hyperthermic intraperitoneal chemotherapy (HIPEC). Koga et al. ${ }^{3}$ from Tottori University, Yonago, Japan successfully performed a randomized controlled trial of prophylactic therapy for peritoneal recurrence of gastric cancer. These investigators used HIPEC with mitomycin C in 1988.

Initially, all efforts to treat established peritoneal metastases were unsuccessful. Dedrick and Flessner ${ }^{4}$ showed that cancer chemotherapy administered into the peritoneal space gained access to the peritoneal surface or to peritoneal cancer nodules only by simple diffusion. The penetration of the drugs into cancerous tissue could be measured only in cell layers. Macroscopic nodules could not be destroyed. Only microscopic disease could be reliably eliminated by intraperitoneal drug delivery. To achieve some level of success in the treatment of established peritoneal metastases with HIPEC, a surgical innovation was required.

This innovation was the peritonectomy procedure $^{5}$ When peritonectomy of anatomic sites involving peritoneal

(C) Society of Surgical Oncology 2017

First Received: 14 September 2017; Published Online: 10 October 2017

P. H. Sugarbaker, MD, FACS, FRCS

e-mail: Paul.Sugarbaker@medstar.net cancer nodules was combined with visceral resections, complete visible clearance of the peritoneal space became a reality. After complete cytoreduction, there was a strong rationale for HIPEC to destroy microscopic residual disease.

The early description of peritonectomy included anterior parietal peritonectomy, right subdiaphragmatic peritonectomy, left subdiaphragmatic peritonectomy, and pelvic peritonectomy. Also, the posterior aspect of the lesser sac could be stripped as a peritonectomy of the omental bursa. ${ }^{6}$ All these dissections were of parietal peritoneum. These parietal peritonectomy procedures were combined with visceral resection of organs that had peritoneal metastases to bring about a complete cytoreductive surgery.

As the surgical technology of cytoreduction evolved over time, involvement of visceral peritoneum, especially the visceral peritoneum of the small bowel, became the most common cause for a suboptimal cytoreductive surgical procedure. The visceral peritoneum of the small bowel was the most common site for treatment failure after cytoreductive surgery plus HIPEC. ${ }^{7}$

Clinical research made it increasingly clear that success in the treatment of peritoneal metastases with cytoreductive surgery and HIPEC required complete cytoreduction of both parietal and visceral peritoneal surfaces. Efforts to remove visceral peritoneum on the small or large bowel mesentery were successful for patients who had a small volume of metastases at this anatomic site. Scissor nodulectomy or electrosurgical evaporation were the surgical technologies discussed for clearing the visceral peritoneum of cancer. ${ }^{8}$ However, all cytoreductive surgeons agreed that the extensively involved surfaces of the small bowel mesentery could not be adequately cytoreduced using this piecemeal approach. 
Deraco et al. ${ }^{9,10}$ were the first to describe the mesenteric peritonectomy procedure for malignant peritoneal mesothelioma patients. Although not all mesothelioma patients could have the small bowel mesentery stripped of its peritoneum using this surgical innovation, selected patients who would have been left with a large volume of malignant peritoneal mesothelioma could leave the operating room with a complete cytoreduction. This complete cytoreduction would be of parietal and visceral peritoneal surfaces.

In this video, Cazauran et al. ${ }^{11}$ illustrate the delicate surgical technology involved in total mesenteric peritonectomy. For a patient with urachal adenocarcinoma and a large volume of peritoneal metastases, they used neoadjuvant systemic chemotherapy with cisplatin, 5-fluorouracil, and docetaxel to bring about a near complete response of the peritoneal metastases. Then, parietal peritonectomies were used to remove disease on parietal peritoneal surfaces. Of course, greater omentectomy was indicated. For this patient, they were able to use the mesenteric peritonectomy to clear the small bowel mesentery peritoneal surfaces of the residual urachal cancer peritoneal dissemination. They skillfully used the electrosurgical scissors to identify a plane beneath the visceral peritoneum but superficial to the delicate vascular supply to the small bowel.

The surgical procedure starts in the midportion of the small bowel mesentery and then works toward the route of the mesentery and also toward the junction of small bowel with its mesentery. The final step of the visceral peritonectomy is amputation of the small bowel peritoneal layer infiltrated by cancer from the visceral peritoneum of the small bowel itself.

This surgical technique for total mesenteric peritonectomy is used only for selected patients. First, these patients must have resection of visible disease at all other anatomic sites within the abdomen and pelvis. Second, only patients with minimally invasive involvement of the visceral peritoneum of the small bowel can be optimally cytoreduced. If nodules are invasive, they will involve the vasculature of the small bowel. Third, those patients with invasive nodules frequently encountered at the junction of small bowel and its mesentery will not be safely or completely resected with this technology. If invasive nodules at the junction of small bowel and small bowel mesentery are to be removed, the portion of small bowel distally must also be resected because it loses its vascular supply with resection of the cancer nodule.

Invasive peritoneal metastases on the surface of the small bowel itself often can be removed with the creation of a seromuscular defect that is then repaired with plicating sutures. If the nodule on a bowel surface is deep, then a full-thickness excision of the bowel wall must occur. ${ }^{8}$
As a future project, long-term function of the small bowel after total mesenteric peritonectomy needs to be critically evaluated. At this writing, Cazauran et al. ${ }^{11}$ report that their patient has remained free of symptomatic peritoneal disease or local recurrence during 6 months of follow-up evaluation. As more patients undergo total mesenteric peritonectomy, the nutritional status and the extent of peritoneal adhesions of these patients need to be evaluated. Are these patients candidates for a reoperative surgery?

This technique is definitely a step forward. I congratulate the surgeons at Lyon-Sud Hospital for showing us that something thought to be impossible in the past is currently a surgical reality. Its clinical efficacy is yet to be determined. However, this technique does promise an improved result with selected patients because it provides a complete cytoreduction that in the past was impossible.

\section{REFERENCES}

1. Yan TD, Black D, Sugarbaker PH, Zhu J, et al. A systematic review and meta-analysis of the randomized controlled trials on adjuvant intraperitoneal chemotherapy for resectable gastric cancer. Ann Surg Oncol. 2007;14:2702-13.

2. Coccolini F, Catena F, Glehen O, Yonemura Y, et al. Effect of intraperitoneal chemotherapy and peritoneal lavage in positive peritoneal cytology in gastric cancer: systematic review and meta-analysis. Eur J Surg Oncol. 2016;42:1261-7.

3. Koga S, Hamazoe R, Maeta M, et al. Prophylactic therapy for peritoneal recurrence of gastric cancer by continuous hyperthermic peritoneal perfusion with mitomycin C. Cancer. 1988;61:232-7.

4. Dedrick RL, Flessner MF. Pharmacokinetic problems in peritoneal drug administration: tissue penetration and surface exposure. J Natl Cancer Inst. 1997;89:480-7.

5. Sugarbaker PH. Peritonectomy procedures. Ann Surg. 1995;221:29-42.

6. De Lima Vazquez V, Sugarbaker PH. Cholecystectomy, lesser omentectomy, and stripping of the omental bursa, a peritonectomy procedure. J Surg Oncol. 2003;84:45-49.

7. Bijelic L, Yan TD, Sugarbaker PH. Failure analysis of recurrent disease following complete cytoreduction and perioperative intraperitoneal chemotherapy in patients with peritoneal carcinomatosis from colorectal cancer. Ann Surg Oncol. 2007; $14: 2281-8$.

8. Bijelic L, Sugarbaker PH. Cytoreduction of the small bowel surfaces. J Surg Oncol. 2008;97:176-9.

9. Deraco M, Baratti D, Kusamura S, et al. Surgical technique of parietal and visceral peritonectomy for peritoneal surface malignancies. J Surg Oncol. 2009;100(4):321-8.

10. Sugarbaker PH, van der Speeten K. An overview of peritonectomy, visceral resections, and therapeutic laparoscopy for peritoneal surface malignancy. In: Sugarbaker PH (ed) Cytoreductive surgery and perioperative chemotherapy for peritoneal surface malignancy. 2nd ed. Cine-Med Publishing, Woodbury, 2017, pp 17-46.

11. Cazauran JB, Lasseur A, Pasquer A, et al. Total mesenteric peritonectomy for peritoneal metastases (with video). Ann Surg Oncol. 2017. doi:10.1245/s10434-017-6099-5. 